\title{
Acute fulminant colon cancer metastasis after renal transplantation
}

\author{
C. T. Lin, T. L. Cha, S. T. Wu and F. P. Chuang \\ Division of Urology. Department of Surgery. Tri-Service General Hospital. National Defense Medical Center. \\ Taipei, Taiwan. Republic of China
}

\begin{abstract}
We report a 52-year-old male with no family history of colonic cancer, who was found to have advanced colonic cancer with metastases two months post renal transplantation. With this case, we highlight the possibility of acute fulminant cancer metastases within short period after renal transplantation and the importance of periodic colorectal cancer screening pre-transplant. To our knowledge, this case is not yet reported in the literature, especially with such presentation of acute fulminant colonic cancer metastases post renal transplantation.
\end{abstract}

Key words: Adenocarcinoma of colon. Colon cancer. Renal transplantation. Immunosuppression. p53 gene mutation.

Lin CT, Cha TL, Wu ST, Chuang FP. Acute fulminant colon cancer metastasis after renal transplantation. Rev Esp Enferm Dig 2010; 102: 447-448.

\section{INTRODUCTION}

Recipients of renal transplants have significantly improved of long-term organ acceptance due to the use of immunosuppressive drugs. Also well documented is a raising risk of developing malignancy after solid-organ transplantation when compared with age-matched controls. Although the association of colonic cancer in postrenal transplant patients is still controversial, Sheil et al. found an increased risk, reporting a risk ratio of 2.6 (1).

Received: 15-03-10.

Accepted: 18-03-10.

Correspondence: Feng-Pin Chuang. Division of Urology. Department of Surgery. Tri-Service General Hospital. No. 325, Cheng-Kung Rd, Sec 2, Taipei 114, Taiwan.e-mail: aarondakimo@yahoo.com.tw
We reported a case of adenocarcinoma of colon with acute fulminant liver and spleen metastases in short-period of two-month after kidney transplantation.

\section{CASE REPORT}

A 52-year-old male presented to our hospital with a one-day history of abdominal cramping pain, watery diarrhea and generalized fatigue. He had just undergone cadaveric renal transplantation two month ago. Abdominal sonography, taken one day post renal transplantation, revealed no remarkable findings. His renal graft function was stable on triple drug therapy including prednisolone, sirolimus, and mycophenolate mofetil (MMF). There is no bowel habit change or weight loss in recent two month after renal transplantation. On admission, his vital signs revealed temperature of $37.8{ }^{\circ} \mathrm{C}$, pulse of $92 / \mathrm{min}$ and blood pressure of $116 / 68 \mathrm{mmHg}$. The blood tests showed white blood count of 4000/ul without predominant segmented neutrophils. Physical examination showed no remarkable findings except epigastric tenderness.

However, abdominal sonography revealed hyperechoic lesions over liver and spleen two months later. Abdominal magnetic resonance image demonstrated lobulated heterogeneous mass about $4.5 \times 5.5 \times 3.6 \mathrm{~cm}$ at the segment 7 and a mild heterogeneous mass about $2.8 \times 2.4$ $\mathrm{cm}$ in the spleen (Fig. 1). Tumor marker studies showed CEA $7.09 \mathrm{ng} / \mathrm{ml}$ and CA199 3385 unit $/ \mathrm{ml}$. Sono-guided needle biopsy of the liver was performed and pathology showed metastatic adenocarcinoma of colon (Fig. 2). Colon fiberoscopy revealed one $1.4 \mathrm{~cm}$ ulcerative mass at splenic flexture, $60 \mathrm{~cm}$ from anal verge and biopsies of the lesion showed adenocarcinoma. Furthermore, immunostaining of paraffin-embedded sections revealed mutations of the p53 gene. Therefore, the patient was referred to oncological section for further chemotherapy with 5-fluorouracil and irinotecan. 

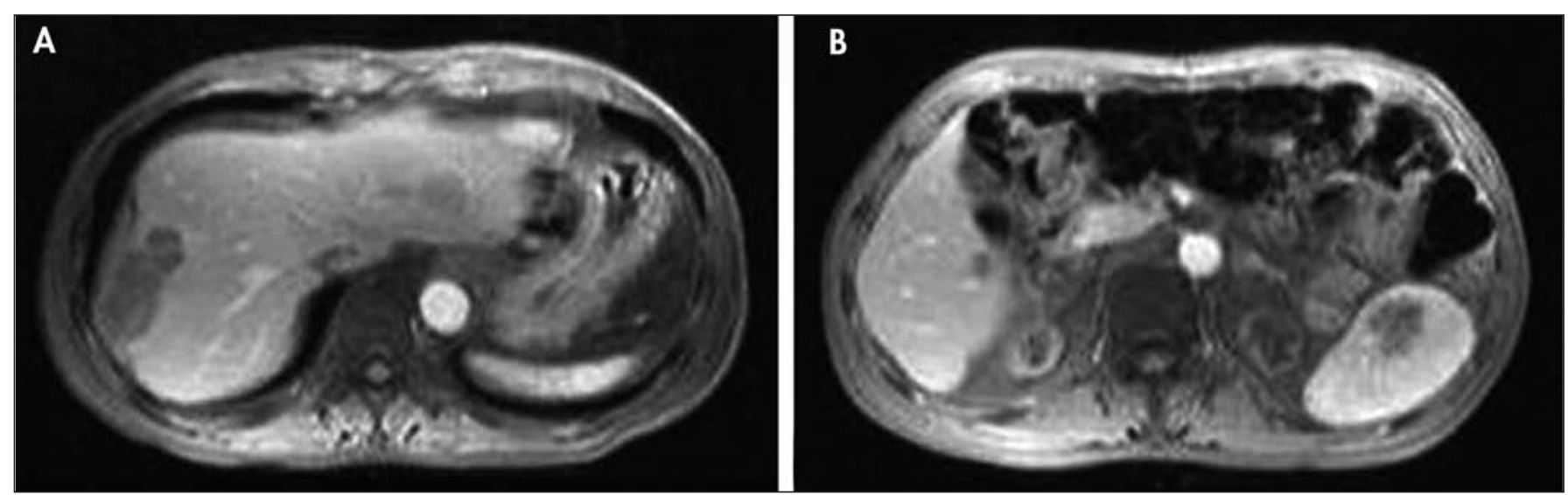

Fig. 1. MRI showed one lobulated heterogeneous mass about $4.5 \times 5.5 \times 3.6 \mathrm{~cm}$ at the segment 7 of liver $(A)$ and one mild heterogeneous mass about $2.8 \times 2.4 \mathrm{~cm}$ in the spleen (B).

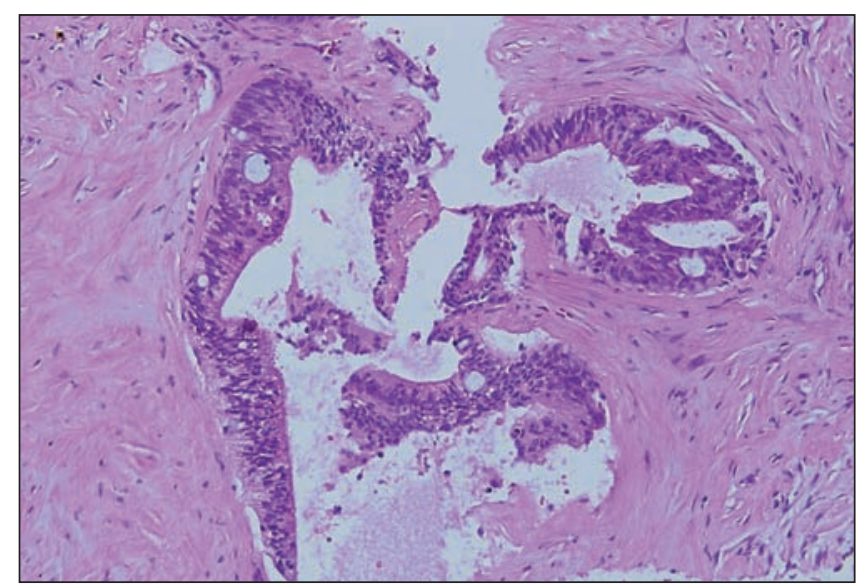

Fig. 2. Pathology showed a picture of metastatic adenocarcinoma accompanied by anaplastic microglands with bizarre nuclei and focal extensive microcalcification, compatible with metastatic adenocarcinoma of colon, moderately differentiated (H\&E stain, 400X).

\section{DISCUSSION}

In the current literature, colorectal cancer after renal transplantation is controversial and the reported time interval from transplant to colon cancer development ranged from 3.8 to 12.3 years (2). The American Society of Transplantation Clinical Practice Guideline recommends the same standard colorectal cancer screening protocol for post-transplant recipients. There is no agreement as to whether colorectal cancer screening guidelines should be altered for post-renal transplant patients, and most transplant registries seldom report whether aggressive or standard postoperative surveillance was administered.

Johnson et al. reported a median age of 58.7 years at the time of diagnosis and concluded that more than $25 \%$ of the at-risk population would have their tumors missed with adherence to current screening guidelines (3). They suggested that those older than age 50, a baseline pre- transplant screening colonoscopy should be obtained along with a follow-up surveillance exam 2 years after transplant. Although colorectal cancer may present earlier and act more aggressively in transplant recipients, more aggressive screening may continue in an at-risk population including two inherited conditions, familial adenomatous polyposis and hereditary nonpolyposis colorectal cancer (4). Besides, p53 gene abnormality and immunosuppressive drugs may have accelerated the tumor onset and development (5).

With improvements in immunosuppressive regimens, malignancies have developed into a frequent long-term complication in kidney recipients. We reported this case of adenocarcinoma of colon with liver and spleen metastases approximately two months after kidney transplantation. While the developed colonic cancer with acute fulminant metastases may be associated with the likelihood of a pre-existing condition which could not be early detected, this aggressive course is believed to be associated with the use of immunosuppressive drugs and p53 gene abnormality. We suggested that more aggressive screening for malignancy studies before renal transplantation, though the age and the timing to initiate screening remain to be determined.

\section{REFERENCES}

1. Sheil AGR. Cancer in dialysis and transplant patients. In: Morris PJ, editor. Kidney transplantation: principles and practice. $4^{\text {th }}$ ed. Philadelphia: WB Saunders; 1994. p. 390-400.

2. Vajdic CM, McDonald SP, McCredie MR, van Leeuwen MT, Stewart $\mathrm{JH}$, Law M, et al. Cancer incidence before and after kidney transplantation. JAMA 2006; 296: 2823-31.

3. Johnson EE, Leverson GE, Pirsch JD, Heise CP. A 30-year analysis of colorectal adenocarcinoma in transplant recipients and proposal for altered screening. J Gastrointest Surg 2007; 11: 272-9.

4. Lynch HT, De la Chapelle A. Hereditary colorectal cancer. N Engl J Med 2003; 348: 919-32.

5. Kitayama T, Marubayashi S, Hayamizu K, Tashiro H, Ohdan H, Ikeda $\mathrm{S}$, et al. Allochronic overlapping malignancies after renal transplantation in a patient with $\mathrm{p} 53$ gene mutation: report of a case. Surg Today 2004; 34: 473-6. 